\title{
Coordinate scaling and adiabatic-connection formulation in density-functional theory
}

\author{
Yan Alexander Wang* \\ Department of Chemistry, University of North Carolina, Chapel Hill, North Carolina 27599
}

(Received 24 February 1997)

\begin{abstract}
Five theorems and one lemma, regarding the limits of density functionals as one or several of the coordinatescaling parameters go to zero or infinity, are proved. A unified description of the coordinate-scaling method and the adiabatic-connection formulation is also introduced. [S1050-2947(97)03308-8]
\end{abstract}

PACS number(s): 31.15.Ew

Modern density-functional theory (DFT) [1] has benefited much from the coordinate-scaling method [2] and the adiabatic-connection formulation [3], which have been used extensively in checking and developing density functionals [1-4]. In the following, a unified formalism of these two theoretical techniques is introduced within the framework of DFT.

Via the constrained-search formulation [5], the Hohenberg-Kohn (HK) universal functional [6] $F_{\lambda}\left[\rho_{\alpha \beta \zeta}^{x y z}\right]$, defined within an extended domain

$$
F_{\lambda}\left[\rho_{\alpha \beta \zeta}^{x y z}\right]=\left\langle\Psi^{\lambda}\left[\rho_{\alpha \beta \zeta}^{x y z}\right]\left|\hat{T}+\lambda \hat{V}_{\mathrm{ee}}\right| \Psi^{\lambda}\left[\rho_{\alpha \beta \zeta}^{x y z}\right]\right\rangle,
$$

always has a minimum [7] for an antisymmetric $N$-electron wave function $\Psi^{\lambda}\left[\rho_{\alpha \beta \zeta}^{x y z}\right]$, with a specific non-negative interelectron interaction coupling constant $\lambda$ and three positive coordinate-scaling parameters $\{\alpha, \beta, \zeta\}$. Here, $\Psi^{\lambda}\left[\rho_{\alpha \beta \zeta}^{x y z}\right]$ generates a coordinate-scaled $N$-representable electron density $\rho_{\alpha \beta \zeta}^{x y z}(\mathbf{r})$, which relates to the original unscaled $v$-representable electron density $\rho(\mathbf{r})$ via [8-12]

$$
\rho_{\alpha \beta \zeta}^{x y z}(x, y, z)=\alpha \beta \zeta \rho(\alpha x, \beta y, \zeta z) .
$$

It can be shown $[1,13]$ that $\Psi^{\lambda}\left[\rho_{\alpha \beta \zeta}^{x y z}\right]$, is an eigenstate (not necessarily the ground state) of the coupled Hamiltonian

$$
\hat{H}_{\lambda}=\hat{T}+\lambda \hat{V}_{\mathrm{ee}}+\hat{V}_{\mathrm{ext}}^{\lambda}
$$

where $\hat{T}, \hat{V}_{\mathrm{ee}}$, and $\hat{V}_{\mathrm{ext}}^{\lambda}$ are the kinetic-energy, the interelectron Coulomb repulsion, and the external potential operators, respectively. Using arguments presented earlier [1,9-12], one can further show that

$$
F_{\lambda}\left[\rho_{\alpha \beta \zeta}^{x y z}\right]=\left\langle\left.\begin{array}{cc}
x y z \\
\alpha \beta \zeta
\end{array} \Psi^{\lambda}\right|_{\alpha \beta \zeta} ^{x y z} \hat{T}+\left.\lambda_{\alpha \beta \zeta}^{x y z} \hat{V}_{\mathrm{ee}}\right|_{\alpha \beta \zeta} ^{x y z} \Psi^{\lambda}\right\rangle,
$$

where ${ }_{\alpha \beta \zeta}^{x y z} \Psi^{\lambda}$ generates $\rho(\mathbf{r})$ directly, minimizes

$$
\left\langle\left.\Psi\right|_{\alpha \beta \zeta} ^{x y z} \hat{T}+\lambda_{\alpha \beta \zeta}^{x y z} \hat{V}_{\mathrm{ee}} \mid \Psi\right\rangle
$$

and is an eigenstate of the coordinate-scaled coupled Hamiltonian

\footnotetext{
*Present address: Department of Chemistry and Biochemistry, University of California, 405 Hilgard Avenue, Los Angeles, CA 90024-1569.
}

$$
{ }_{\alpha \beta \zeta}^{x y z} \hat{H}_{\lambda}={ }_{\alpha \beta \zeta}^{x y z} \hat{T}+\lambda_{\alpha \beta \zeta}^{x y z} \hat{V}_{\mathrm{ee}}+{ }_{\alpha \beta \zeta}^{x y z} \hat{V}_{\mathrm{ext}}^{\lambda} .
$$

Here ${ }_{\alpha \beta \zeta}^{x y z} \hat{T}, \underset{\alpha \beta \zeta}{x y z} \hat{V}_{\mathrm{ee}}$, and ${ }_{\alpha \beta \zeta}^{x y z} \hat{V}_{\mathrm{ext}}^{\lambda}$, are coordinate scaled as

$$
\begin{aligned}
& \underset{\substack{x \beta \zeta \\
\alpha \beta \zeta}}{x}=-\frac{1}{2} \sum_{i=1}^{N}\left(\alpha^{2} \frac{\partial^{2}}{\partial x_{i}^{2}}+\beta^{2} \frac{\partial^{2}}{\partial y_{i}^{2}}+\zeta^{2} \frac{\partial^{2}}{\partial z_{i}^{2}}\right), \\
& \underset{\alpha \beta \zeta}{x y z} \hat{V}_{\mathrm{ee}}=\sum_{i>j}^{N} 1 /\left(\frac{x_{i j}^{2}}{\alpha^{2}}+\frac{y_{i j}^{2}}{\beta^{2}}+\frac{z_{i j}^{2}}{\zeta^{2}}\right)^{1 / 2}, \\
& { }_{\alpha \beta \zeta}^{x y z} \hat{V}_{\mathrm{ext}}^{\lambda}=\hat{V}_{\mathrm{ext}}^{\lambda}\left(x_{i} / \alpha, y_{i} / \beta, z_{i} / \zeta\right),
\end{aligned}
$$

with $x_{i j}=x_{i}-x_{j}, y_{i j}=y_{i}-y_{j}$, and $z_{i j}=z_{i}-z_{j}$,

In the spirit of the Kohn-Sham (KS) theory [14],

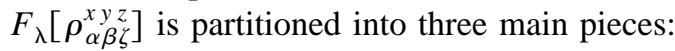

$$
F_{\lambda}\left[\rho_{\alpha \beta \zeta}^{x y z}\right]=T_{s}\left[\rho_{\alpha \beta \zeta}^{x y z}\right]+\lambda J\left[\rho_{\alpha \beta \zeta}^{x y z}\right]+\lambda E_{\mathrm{xc}}^{\lambda}\left[\rho_{\alpha \beta \zeta}^{x y z}\right],
$$

where $T_{s}\left[\rho_{\alpha \beta \zeta}^{x y z}\right]$ is the coordinate-scaled noninteracting $(\lambda$ $=0)$ kinetic-energy functional, $J\left[\rho_{\alpha \beta \zeta}^{x y z}\right]$ is the coordinatescaled classical interelectron Coulomb repulsion functional, and $E_{\mathrm{xc}}^{\lambda}\left[\rho_{\alpha \beta \zeta}^{x y z}\right]$ is the coordinate-scaled exchange-correlation functional. Following an earlier work by Levy and Perdew [8], one can decompose $E_{\mathrm{xc}}^{\lambda}\left[\rho_{\alpha \beta \zeta}^{x y z}\right]$ into two components:

$$
E_{\mathrm{xc}}^{\lambda}\left[\rho_{\alpha \beta \zeta}^{x y z}\right]=E_{x}\left[\rho_{\alpha \beta \zeta}^{x y z}\right]+E_{c}^{\lambda}\left[\rho_{\alpha \beta \zeta}^{x y z}\right]
$$

namely, the $\lambda$-dependent coordinate-scaled exchange func-

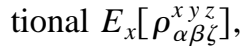

$$
\begin{aligned}
E_{x}\left[\rho_{\alpha \beta \zeta}^{x y} z\right. & =\left\langle\left.\left.\begin{array}{cc}
x y z \\
\alpha \beta \zeta
\end{array} \Psi^{\lambda=0}\right|_{\alpha \beta \zeta} ^{x y z} \hat{V}_{\mathrm{ee}}\right|_{\alpha \beta \zeta} ^{x y z} \Psi^{\lambda=0}\right\rangle-J\left[\rho_{\alpha \beta \zeta}^{x y z}\right] \\
& =V_{\mathrm{ee}}^{0}\left[\rho_{\alpha \beta \zeta}^{x y z}\right]-J\left[\rho_{\alpha \beta \zeta}^{x y z}\right]
\end{aligned}
$$

and the $\lambda$-independent coordinate-scaled correlation functional $E_{c}^{\lambda}\left[\begin{array}{c}x y z \\ \alpha \beta \zeta\end{array}\right]$,

$$
E_{c}^{\lambda}\left[\rho_{\alpha \beta \zeta}^{x y z}\right]=(1 / \lambda) T_{c}^{\lambda}\left[\rho_{\alpha \beta \zeta}^{x y z}\right]+V_{c}^{\lambda}\left[\rho_{\alpha \beta \zeta}^{x y z}\right],
$$

where

$$
\begin{aligned}
T_{c}^{\lambda}\left[\rho_{\alpha \beta \zeta}^{x y z}\right]= & \left\langle\left.\left.\begin{array}{c}
x y z \\
\alpha \beta \zeta
\end{array} \Psi^{\lambda}\right|_{\alpha \beta \zeta} ^{x y z} \hat{T}\right|_{\alpha \beta \zeta} ^{x y z} \Psi^{\lambda}\right\rangle \\
& -\left\langle\left.\left.\begin{array}{c}
x y z \\
\alpha \beta \zeta
\end{array} \Psi^{\lambda=0}\right|_{\alpha \beta \zeta} ^{x y z} \hat{T}\right|_{\alpha \beta \zeta} ^{x y z} \Psi^{\lambda=0}\right\rangle \\
= & T^{\lambda}\left[\rho_{\alpha \beta \zeta}^{x y z}\right]-T_{s}\left[\rho_{\alpha \beta \zeta}^{x y z}\right]
\end{aligned}
$$

and 


$$
\begin{aligned}
V_{c}^{\lambda}\left[\rho_{\alpha \beta \zeta}^{x y z}\right] & =\left\langle\left.\left.\begin{array}{c}
x y z \\
\alpha \beta \zeta
\end{array} \Psi^{\lambda}\right|_{\alpha \beta \zeta} ^{x y z} \hat{V}_{\mathrm{ee}}\right|_{\alpha \beta \zeta} ^{x y z} \Psi^{\lambda}\right\rangle-V_{\mathrm{ee}}^{0}\left[\rho_{\alpha \beta \zeta}^{x y z}\right] \\
& =V_{\mathrm{ee}}^{\lambda}\left[\rho_{\alpha \beta \zeta}^{x y z}\right]-V_{\mathrm{ee}}^{0}\left[\rho_{\alpha \beta \zeta}^{x y z}\right] .
\end{aligned}
$$

At $\lambda=0, F_{\lambda=0}\left[\rho_{\alpha \beta \zeta}^{x y z}\right]$ reduces to $T_{s}\left[\rho_{\alpha \beta \zeta}^{x y z}\right], \underset{\alpha \beta \zeta}{x y z} \Psi^{\lambda=0}$ becomes a single Slater determinant $\begin{gathered}x y z \\ \alpha \beta \zeta\end{gathered} \Phi$ made from the first$N$ KS orbitals $\left\{\begin{array}{c}x y z \\ \alpha \beta \zeta\end{array} \phi_{i}^{\mathrm{KS}}\right\}$, and $\begin{gathered}x y z \\ \alpha \beta \xi\end{gathered} \hat{V}_{\mathrm{ext}}^{\lambda=0}$ turns into the KS effective potential ${ }_{\alpha \beta \zeta}^{x y z} \hat{V}_{\mathrm{eff}}^{\mathrm{KS}}[14]$.

Without loss of generality, the coordinate-scaling parameters $\{\beta, \zeta\}$ will be assumed to be analytic functions of $\alpha$. Unless otherwise noted hereafter, a dummy functional $G_{c}^{\lambda}\left[\rho_{\alpha \beta \zeta}^{x y z}\right]$ will be used to denote $E_{c}^{\lambda}\left[\rho_{\alpha \beta \zeta}^{x y z}\right], V_{c}^{\lambda}\left[\rho_{\alpha \beta \zeta}^{x y z}\right]$, or $T_{c}^{\lambda}\left[\rho_{\alpha \beta \zeta}^{x y z}\right]$, and another dummy functional $H\left[\rho_{\alpha \beta \zeta}^{x y z}\right]$ will be used to denote $E_{x}\left[\rho_{\alpha \beta \zeta}^{x y z}\right], J\left[\rho_{\alpha \beta \zeta}^{x y z}\right]$, or $V_{\mathrm{ee}}^{0}\left[\rho_{\alpha \beta \zeta}^{x y z}\right]$.

The definitions of ${ }_{\alpha \beta \zeta}^{x y z} \Psi^{\lambda}$ and ${ }_{\alpha \beta \zeta}^{x y z} \Phi$ ensure that

$$
V_{c}^{\lambda}\left[\rho_{\alpha \beta \zeta}^{x y z}\right] \leqslant E_{c}^{\lambda}\left[\rho_{\alpha \beta \zeta}^{x y z}\right] \leqslant 0 \leqslant T_{c}^{\lambda}\left[\rho_{\alpha \beta \zeta}^{x y z}\right] \leqslant-\lambda V_{c}^{\lambda}\left[\rho_{\alpha \beta \zeta}^{x y z}\right] .
$$

Using Eqs. (13) and (16), one arrives at a lemma.

Lemma: If an analytic function of $\alpha, f_{0}(\alpha)$, satisfies

$$
\lim _{\alpha}\left(f_{0}(\alpha) V_{c}^{\lambda}\left[\rho_{\alpha \beta \zeta}^{x y z}\right]\right)=0
$$

then it also satisfies

$$
\lim _{\alpha}\left(f_{0}(\alpha) G_{c}^{\lambda}\left[\rho_{\alpha \beta \zeta}^{x y z}\right]\right)=0 .
$$

Similarly, if an analytic function of $\alpha, f_{1}(\alpha)$, satisfies

$$
\lim _{\alpha}\left(f_{1}(\alpha) V_{c}^{\lambda}\left[\rho_{\alpha \beta \zeta}^{x y z}\right]\right)=\text { const }
$$

then it also satisfies

$$
\lim _{\alpha}\left(f_{1}(\alpha) G_{c}^{\lambda}\left[\rho_{\alpha \beta \zeta}^{x y z}\right]\right)=\text { const }
$$

In principle, for an analytic function $f_{2}(\alpha)$, $\lim _{\alpha}\left(f_{2}(\alpha) E_{c}^{\lambda}\left[\begin{array}{c}x y z \\ \alpha \beta \zeta\end{array}\right]\right)$ can be zero, a finite constant, or infinity when $\lim _{\alpha}\left(f_{2}(\alpha) T_{c}^{\lambda}\left[\rho_{\alpha \beta \zeta}^{x y z}\right]\right)$ and $\lim _{\alpha}\left(f_{2}(\alpha) V_{c}^{\lambda}\left[\rho_{\alpha \beta \zeta}^{x y z}\right]\right)$ diverge. However, there is a great chance practically for them all to be divergent at the same time. This lemma suggests that one only need to pay attention to the limit of $f(\alpha) V_{c}^{\lambda}\left[\rho_{\alpha \beta \zeta}^{x y z}\right]$ in order to figure out the limits of $f(\alpha) E_{c}^{\lambda}\left[\rho_{\alpha \beta \zeta}^{x y z}\right]$ and $f(\alpha) T_{c}^{\lambda}\left[\rho_{\alpha \beta \zeta}^{x y z}\right]$ for an analytic function $f(\alpha)$. The two special cases $\alpha \rightarrow 0$ and $\alpha \rightarrow \infty$ will be considered below.

First, as $\alpha \rightarrow 0,\{\alpha, \beta, \zeta\}$ are assumed to be $\alpha \leqslant \beta \leqslant \zeta$, and $\xi \equiv\left(\alpha / \zeta^{2}\right)$ is assumed to be 0 :

$$
\lim _{\alpha \rightarrow 0} \xi=0 .
$$

Then the interelectron Coulomb interaction operator in Eq. (8),

$$
{ }_{\alpha \beta \zeta}^{x y z} \hat{V}_{\mathrm{ee}}=\zeta^{2} \xi \sum_{i>j}^{N} 1 /\left(x_{i j}^{2}+\frac{\alpha^{2}}{\beta^{2}} y_{i j}^{2}+\frac{\alpha^{2}}{\zeta^{2}} z_{i j}^{2}\right)^{1 / 2},
$$

can be treated as a perturbation to the kinetic-energy operator in Eq. (7),

$$
{ }_{\alpha \beta \zeta}^{x y z} \hat{T}=-\frac{1}{2} \zeta^{2} \sum_{i=1}^{N}\left(\frac{\partial^{2}}{\partial z_{i}^{2}}+\frac{\beta^{2}}{\zeta^{2}} \frac{\partial^{2}}{\partial y_{i}^{2}}+\frac{\alpha^{2}}{\zeta^{2}} \frac{\partial^{2}}{\partial x_{i}^{2}}\right) .
$$

Also, physical intuition indicates that ${ }_{\alpha \beta \zeta}^{x y z} \hat{V}_{\text {ext }}^{\lambda}$ converges to ${ }_{\alpha \beta \zeta}^{x y z} \hat{V}_{\text {eff }}^{\text {KS }}$ as $O\left(\zeta^{2} \xi\right)$; otherwise, either ${ }_{\alpha \beta \zeta}^{x y z} \hat{V}_{\text {ext }}^{\lambda}$ would have a nonphysical residue for a virtually zero ${ }_{\alpha \beta \zeta}^{x y z} \hat{V}_{\text {ee }}$, or ${ }_{\alpha \beta \zeta}^{x y z} \hat{V}_{\text {ee }}$ would have a nonphysical residue for an almost converged ${ }_{\alpha \beta \zeta}^{x y z} \hat{V}_{\text {ext }}^{\lambda}$. Thus one can expand ${ }_{\alpha \beta \zeta}^{x y z} \Psi^{\lambda}$ as a power series from ${ }_{\alpha \beta \zeta}^{x y z} \Phi$ with the parameter $\xi$,

$$
\underset{\alpha \beta \zeta}{x y z} \Psi^{\lambda}={ }_{\alpha \beta \zeta}^{x y z} \Phi+\sum_{n=1}^{\infty}\left(\begin{array}{c}
x y z \\
\alpha \beta \zeta
\end{array} \Phi_{n}^{\lambda}\right) \xi^{n}
$$

where ${ }_{\alpha \beta \zeta}^{x y z} \Phi_{n}^{\lambda}$ is the $n$ th-order wave function. Inserting Eq. (24) into Eq. (15) yields

$$
V_{c}^{\lambda}\left[\rho_{\alpha \beta \zeta}^{x y z}\right]=\alpha \sum_{n=1}^{\infty} V_{n}^{\lambda}\left[\rho_{\alpha \beta \zeta}^{x y z}\right] \xi^{n},
$$

where $V_{n}^{\lambda}\left[\rho_{\alpha \beta \zeta}^{x y z}\right]$ is the $n$ th-order correlation potential energy. From Eq. (25) and the lemma, one arrives at the following theorem.

Theorem 1: Assume that as $\alpha \rightarrow 0, \alpha \leqslant \beta \leqslant \zeta$, and $\lim _{\alpha} \xi$ $=0$. If an analytic function of $\alpha, f_{0}(\alpha)$, satisfies

$$
\lim _{\alpha \rightarrow 0}\left(f_{0}(\alpha) \alpha \xi\right)=0
$$

then it also satisfies

$$
\lim _{\alpha \rightarrow 0}\left(f_{0}(\alpha) G_{c}^{\lambda}\left[\rho_{\alpha \beta \zeta}^{x y z}\right]\right)=0 .
$$

Similarly, if an analytic function of $\alpha, f_{1}(\alpha)$, satisfies

$$
\lim _{\alpha \rightarrow 0}\left(f_{1}(\alpha) \alpha \xi\right)=\text { const }
$$

then it also satisfies

$$
\lim _{\alpha \rightarrow 0}\left(f_{1}(\alpha) G_{c}^{\lambda}\left[\rho_{\alpha \beta \zeta}^{x y z}\right]\right)=\text { const } .
$$

A simple choice for $f_{1}(\alpha)$ in Eq. (28) is just $1 /(\alpha \xi)$.

When Eq. (21) is invalid, the interelectron Coulomb interaction operator in Eq. (22) can no longer be treated as a perturbation to the kinetic-energy operator in Eq. (23), and hence Eqs. (24) and (25) are also invalid. However, one can still rely on the lemma, and conclude the following theorem, since this time, $V_{c}^{\lambda}\left[\rho_{\alpha \beta \zeta}^{x y z}\right]$ is simply $O(\alpha)$.

Theorem 2: Assume that as $\alpha \rightarrow 0, \alpha \leqslant \beta \leqslant \zeta$, and $\lim _{\alpha}|\xi|>0$. If an analytic function of $\alpha, f_{0}(\alpha)$, satisfies

$$
\lim _{\alpha \rightarrow 0}\left(f_{0}(\alpha) \alpha\right)=0
$$

then it also satisfies

$$
\lim _{\alpha \rightarrow 0}\left(f_{0}(\alpha) G_{c}^{\lambda}\left[\rho_{\alpha \beta \zeta}^{x y z}\right]\right)=0 .
$$


Similarly, if an analytic function of $\alpha, f_{1}(\alpha)$, satisfies

$$
\lim _{\alpha \rightarrow 0}\left(f_{1}(\alpha) \alpha\right)=\text { const }
$$

then is also satisfies

$$
\lim _{\alpha \rightarrow 0}\left(f_{1}(\alpha) G_{c}^{\lambda}\left[\rho_{\alpha \beta \zeta}^{x y z}\right]\right)=\text { const } .
$$

Second, as $\alpha \rightarrow \infty,\{\alpha, \beta, \zeta\}$ are assumed to be $\alpha \geqslant \beta \geqslant \zeta$, and $\eta \equiv\left(\zeta / \alpha^{2}\right)$ is always 0 :

$$
\lim _{\alpha \rightarrow \infty} \eta=0 .
$$

Then the interelectron Coulomb interaction operator in Eq. (8),

$$
{ }_{\alpha \beta \zeta}^{x y z} \hat{V}_{\mathrm{ee}}=\alpha^{2} \eta \sum_{i>j}^{N} 1 /\left(z_{i j}^{2}+\frac{\zeta^{2}}{\beta^{2}} y_{i j}^{2}+\frac{\zeta^{2}}{\alpha^{2}} x_{i j}^{2}\right)^{1 / 2},
$$

can be regarded as a perturbation to the kinetic-energy operator in Eq. (7),

$$
\underset{\alpha \beta \zeta}{x y z} \hat{T}=-\frac{1}{2} \alpha^{2} \sum_{i=1}^{N}\left(\frac{\partial^{2}}{\partial x_{i}^{2}}+\frac{\beta^{2}}{\alpha^{2}} \frac{\partial^{2}}{\partial y_{i}^{2}}+\frac{\zeta^{2}}{\alpha^{2}} \frac{\partial^{2}}{\partial z_{i}^{2}}\right) .
$$

Based on a reason similar to that above, $\begin{gathered}x y z \\ \alpha \beta \zeta\end{gathered} V_{\text {ext }}^{\lambda}$ converges to ${ }_{\alpha \beta \zeta}^{x y z} V_{\mathrm{eff}}^{\mathrm{KS}}$ as $O\left(\alpha^{2} \eta\right)$. Thus one can expand ${ }_{\alpha \beta \zeta}^{x y z} \Psi^{\lambda}$ as a power series from ${ }_{\alpha \beta \zeta}^{x y z} \Phi$ with the parameter $\eta$,

$$
{ }_{\alpha \beta \zeta}^{x y z} \Psi^{\lambda}={ }_{\alpha \beta \zeta}^{x y z} \Phi+\sum_{n=1}^{\infty}\left(\begin{array}{c}
x y z \\
\alpha \beta \zeta
\end{array} \Phi_{\lambda}^{n}\right) \eta^{n}
$$

where ${ }_{\alpha \beta \zeta}^{x y z} \Phi_{\lambda}^{n}$ is the $n$ th-order wave function. Inserting Eq. (37) into Eq. (15) yields

$$
V_{c}^{\lambda}\left[\rho_{\alpha \beta \zeta}^{x y z}\right]=\zeta \sum_{n=1}^{\infty} V_{\lambda}^{n}\left[\rho_{\alpha \beta \zeta}^{x y z}\right] \eta^{n}
$$

where $V_{\lambda}^{n}\left[\rho_{\alpha \beta \zeta}^{x y z}\right]$ is the $n$ th-order correlation potential energy. From Eq. (38) and the lemma, one arrives at the following theorem.

Theorem 3: Assume that as $\alpha \rightarrow \infty, \alpha \geqslant \beta \geqslant \zeta$, and $\lim _{\alpha} \eta=0$. If an analytic function of $\alpha, g_{0}(\alpha)$, satisfies

$$
\lim _{\alpha \rightarrow \infty}\left(g_{0}(\alpha) \zeta \eta\right)=0
$$

then it also satisfies

$$
\lim _{\alpha \rightarrow \infty}\left(g_{0}(\alpha) G_{c}^{\lambda}\left[\rho_{\alpha \beta \zeta}^{x y z}\right]\right)=0
$$

Similarly, if an analytic function of $\alpha, g_{1}(\alpha)$, satisfies

$$
\lim _{\alpha \rightarrow \infty}\left(g_{1}(\alpha) \zeta \eta\right)=\text { const }
$$

then it also satisfies

$$
\lim _{\alpha \rightarrow \infty}\left(g_{1}(\alpha) G_{c}^{\lambda}\left[\rho_{\alpha \beta \zeta}^{x y z}\right]\right)=\text { const }
$$

TABLE I. Some popular choices for $\{\alpha, \beta, \zeta\}$ and their associated $f_{0}(\alpha), f_{1}(\alpha), g_{0}(\alpha)$, and $g_{1}(\alpha)$ functions in theorems $1-3$. $^{\mathrm{a}-\mathrm{c}}$

\begin{tabular}{lcccc}
\hline \hline$\{\alpha, \beta, \zeta\}$ & $f_{0}(\alpha)$ & $f_{1}(\alpha)$ & $g_{0}(\alpha)$ & $g_{1}(\alpha)$ \\
\hline$\left\{\alpha^{k}, \alpha^{k}, \alpha^{k}\right\}$ & $\alpha^{-p}(p<k)$ & $\alpha^{-k}$ & $\alpha^{p}(p<0)$ & 1 \\
$\left\{\alpha^{k}, \alpha^{m}, \alpha^{n}\right\}$ & $\alpha^{-p}(p<q)$ & $\alpha^{-q}$ & $\alpha^{p}(p<q)$ & $\alpha^{q}$ \\
\hline \hline
\end{tabular}

${ }^{\mathrm{a}}$ Some of these cases can be found in Refs. [2, 8-10], but with $\lambda$ $=1$.

${ }^{\mathrm{b}}$ The coordinate-scaling parameters $\{\alpha, \beta, \zeta\}$ are assumed as $\left\{\alpha^{k}, \alpha^{m}, \alpha^{n}\right\}$, such that $k \geqslant m \geqslant n$ and $q=2(k-n)>0$.

${ }^{\mathrm{c}}$ These $f_{0}(\alpha), f_{1}(\alpha), g_{0}(\alpha)$, and $g_{1}(\alpha)$ functions are taken as simple powers of $\alpha$.

A simple choice for $g_{1}(\alpha)$ in Eq. (41) is just $1 /(\zeta \eta)$.

Many requirements for $E_{c}^{\lambda}\left[\rho_{\alpha \beta \zeta}^{x y z}\right]$ obtained earlier [2,810] are applications of these three theorems. Especially, at $\lambda=1$, the constants in Eqs. (36b) and (43b) of Ref. [10] are identified as zero:

$$
\lim _{\alpha \rightarrow \infty}\left(\alpha E_{c}\left[\rho_{\alpha 11}^{x y z}\right]\right)=\lim _{\alpha \rightarrow 0}\left(E_{c}\left[\rho_{\alpha \alpha 1 / \alpha}^{x y z}\right] / \alpha^{2}\right)=0
$$

Following the description of the lemma and theorems 1-3, one can easily carry out the same analysis for more complex cases as those collected in Table I. It is also necessary to emphasize that the present discussion differs from an earlier work [10] in two ways: first, Eq. (16) and the lemma have been observed throughout the derivation, and, second, the convergence property of ${ }_{\alpha \beta \zeta}^{x y z} V_{\text {ext }}^{\lambda}$ has been taken into consideration in the perturbation expansions of Eqs. (24) and (37).

The same kind of analysis can also be carried out for $E_{x}\left[\rho_{\alpha \beta \zeta}^{x y z}\right]$. Using the KS orbitals, one can readily simplify Eq. (12) to

$E_{x}\left[\rho_{\alpha \beta \zeta}^{x y z}\right]=-\frac{1}{2}\left\langle\left.||_{\alpha \beta \zeta}^{x y z} \gamma^{\mathrm{KS}}\left(\mathbf{r}_{1}, \mathbf{r}_{2}\right)\right|^{2} /\left(\frac{x_{12}^{2}}{\alpha^{2}}+\frac{y_{12}^{2}}{\beta^{2}}+\frac{z_{12}^{2}}{\zeta^{2}}\right)^{1 / 2}\right)$,

where ${ }_{\alpha \beta \zeta}^{x y z} \gamma^{\mathrm{KS}}\left(\mathbf{r}_{1}, \mathbf{r}_{2}\right)$ is the KS first-order reduced density matrix:

$$
\underset{\alpha \beta \zeta}{x y z} \gamma^{\mathrm{KS}}\left(\mathbf{r}_{1}, \mathbf{r}_{2}\right)=\sum_{i=1}^{N}\left[\begin{array}{c}
x y z \\
\alpha \beta \zeta
\end{array} \phi_{i}^{\mathrm{KS}}\left(\mathbf{r}_{2}\right)\right]^{*} \times\left[\begin{array}{c}
x y \beta \zeta \\
\alpha \beta \zeta
\end{array} \phi_{i}^{\mathrm{KS}}\left(\mathbf{r}_{1}\right)\right] .
$$

Invoking Roothaan's arguments [15], one knows that

$$
-J\left[\rho_{\alpha \beta \zeta}^{x y z}\right] \leqslant E_{x}\left[\rho_{\alpha \beta \zeta}^{x y z}\right] \leqslant 0
$$

Also, a direct observation of the positivity of $V_{\mathrm{ee}}^{0}\left[\rho_{\alpha \beta \zeta}^{x y z}\right]$ from Eq. (12) and the negativity of $E_{x}\left[\rho_{\alpha \beta \zeta}^{x y z}\right]$ from Eq. (44) leads to a much simpler derivation of the same result.

Theorem 4: Assume that, as $\alpha \rightarrow 0, \alpha \leqslant \beta \leqslant \zeta$,

$$
\lim _{\alpha \rightarrow 0}(\beta / \alpha)=C_{\beta \alpha} \quad \text { and } \quad \lim _{\alpha \rightarrow 0}(\zeta / \alpha)=C_{\zeta \alpha}
$$

If an analytic function of $\alpha, h(\alpha)$, satisfies 


$$
\lim _{\alpha \rightarrow 0}(h(\alpha) \alpha)=C_{h \alpha},
$$

then it also satisfies

$$
\lim _{\alpha \rightarrow 0}\left(h(\alpha) H\left[\rho_{\alpha \beta \zeta}^{x y z}\right]\right)=C_{h \alpha} H\left[\rho_{1 C_{\beta \alpha} C_{\zeta \alpha}}^{x y}\right] .
$$

Theorem 5: Assume that as $\alpha \rightarrow \infty, \alpha \geqslant \beta \geqslant \zeta$,

$$
\lim _{\alpha \rightarrow \infty}(\alpha / \beta)=C_{\alpha \beta} \quad \text { and } \quad \lim _{\alpha \rightarrow \infty}(\alpha / \zeta)=C_{\alpha \zeta} .
$$

If an analytic function of $\alpha, k(\alpha)$, satisfies

$$
\lim _{\alpha \rightarrow \infty}(k(\alpha) \zeta)=C_{k \zeta}
$$

then it also satisfies

$$
\lim _{\alpha \rightarrow \infty}\left(k(\alpha) H\left[\rho_{\alpha \beta \zeta}^{x y z}\right]\right)=C_{k \zeta} H\left[\rho_{C_{\alpha \zeta}^{x y} C_{\alpha \beta} 1}\right] .
$$

The proofs of theorems 4 and 5 from Eqs. (44) and (46) are elementary, and hence are omitted here. Also, Eqs. (49) and (52) automatically satisfy the general inequality in Eq. (46). With these five theorems and the lemma, one can then easily discuss the limits of $f(\alpha) E_{\mathrm{xc}}^{\lambda}\left[\rho_{\alpha \beta \zeta}^{x y z}\right]$ for any analytic function $f(\alpha)$.

Finally, it is worthwhile to point out the intrinsic equivalence $[16,17]$ between the uniform coordinate scaling $[2,8]$ and the adiabatic-connection formulation [3]. In Eqs. (1) and (4), after setting $\alpha=\beta=\zeta$, one has an identity

$$
F_{\lambda}\left[\rho_{\alpha \alpha \alpha}^{x y z}\right]=\alpha^{2} F_{\lambda / \alpha}[\rho]
$$

From Eqs. (10)-(15) and (53), one can further derive the following identities:

$$
\begin{array}{cc}
T_{s}\left[\rho_{\alpha \alpha \alpha}^{x y z}\right]=\alpha^{2} T_{s}[\rho], & H\left[\rho_{\alpha \alpha \alpha}^{x y z}\right]=\alpha H[\rho], \\
K^{\lambda}\left[\rho_{\alpha \alpha \alpha}^{x y z}\right]=\alpha^{2} K^{\lambda / \alpha}[\rho], & L^{\lambda}\left[\rho_{\alpha \alpha \alpha}^{x y z}\right]=\alpha L^{\lambda / \alpha}[\rho],
\end{array}
$$

where the dummy functional $K^{\lambda}\left[\rho_{\alpha \alpha \alpha}^{x y z}\right]$ denotes $T_{c}^{\lambda}\left[\rho_{\alpha \alpha \alpha}^{x y z}\right]$ or $T^{\lambda}\left[\rho_{\alpha \alpha \alpha}^{x y z}\right]$, and the dummy functional $L^{\lambda}\left[\rho_{\alpha \alpha \alpha}^{x y z}\right]$ denotes $E_{\mathrm{xc}}^{\lambda}\left[\rho_{\alpha \alpha \alpha}^{x y z}\right], E_{c}^{\lambda}\left[\rho_{\alpha \alpha \alpha}^{x y z}\right], V_{c}^{\lambda}\left[\rho_{\alpha \alpha \alpha}^{x y z}\right]$, or $V_{\mathrm{ee}}^{\lambda}\left[\rho_{\alpha \alpha \alpha}^{x y z}\right]$. In Eq. (54), the first two identities were first introduced in Ref. [8], and the identity for $E_{c}\left[\rho_{\alpha \alpha \alpha}^{x y z}\right]$ was derived in Refs. [16,17]. Further, the general identity for $E_{c}^{\lambda}\left[\rho_{\alpha \alpha \alpha}^{x y z}\right]$ in Eq. (54) has been used to generalize [18] the original Levy-Perdew equation $[8,17]$ to

$$
T_{c}^{\lambda}[\rho]=-\lambda E_{c}^{\lambda}[\rho]+\lambda\left(\frac{\partial E_{c}^{\lambda}\left[\rho_{\alpha \alpha \alpha}^{x y z}\right]}{\partial \alpha}\right)_{\alpha=1},
$$

from the identity [19]

$$
T_{c}^{\lambda}[\rho]=-\lambda^{2} \frac{d E_{c}^{\lambda}[\rho]}{d \lambda} .
$$

This research was supported by a grant from the National Science Foundation to the University of North Carolina at Chapel Hill. Encouragement and help from Professor Robert G. Parr are gratefully appreciated.
[1] R. G. Parr and W. Yang, Density-Functional Theory of Atoms and Molecules (Oxford University Press, New York, 1989).

[2] For a comprehensive review, see M. Levy, in Density Functional Theory, edited by E. K. U. Gross and R. M. Dreizler (Plenum, New York, 1995), p. 11, and references therein.

[3] J. Harris and R. O. Jones, J. Phys. F 4, 1170 (1974); O. Gunnarsson and B. I. Lundqvist, Phys. Rev. B 13, 4274 (1976); D. C. Langreth and J. P. Perdew, ibid. 21, 5469 (1980); J. Harris, Phys. Rev. A 29, 1648 (1984).

[4] For example, M. Levy, N. H. March, and N. C. Handy, J. Chem. Phys. 104, 1989 (1996).

[5] J. K. Percus, Int. J. Quantum Chem. 13, 89 (1978); M. Levy, Proc. Natl. Acad. Sci. USA 76, 6062 (1979).

[6] P. Hohenberg and W. Kohn, Phys. Rev. 136, B864 (1964).

[7] E. H. Lieb, Int. J. Quantum Chem. 24, 243 (1983).

[8] M. Levy and J. P. Perdew, Phys. Rev. A 32, 2010 (1985).

[9] H. Ou-Yang and M. Levy, Phys. Rev. A 42, 155 (1990).

[10] A. Görling and M. Levy, Phys. Rev. A 45, 1509 (1992).
[11] M. Levy, W. Yang, and R. G. Parr, J. Chem. Phys. 83, 2334 (1985).

[12] M. Levy and H. Ou-Yang, Phys. Rev. A 42, 651 (1990).

[13] K. F. Freed and M. Levy, J. Chem. Phys. 77, 396 (1982); J. P. Perdew and M. Levy, Phys. Rev. B 31, 6264 (1985).

[14] W. Kohn and L. J. Sham, Phys. Rev. 140, A1133 (1965).

[15] C. C. J. Roothaan, Rev. Mod. Phys. 23, 69 (1951).

[16] M. Levy in Single-Particle Density in Physics and Chemistry, edited by N. H. March and B. M. Deb (Academic, London, 1987); W. Yang, in Density Matrices and Density Functionals, edited by R. Erdahl and V. H. Smith, Jr. (Reidel, Boston, 1987).

[17] A. Görling and M. Levy, Phys. Rev. B 47, 13105 (1993).

[18] Y. A. Wang, Chem. Phys. Lett. 268, 76 (1997); 271, 386(E) (1997).

[19] A. Savin, Phys. Rev. A 52, 1805 (1995); S. Liu and R. G. Parr, ibid. 53, 2211 (1996). 